\title{
Factors impacting on menstrual hygiene and their implications for health promotion
}

\author{
Anne Mutunda Lahme, Ruth Stern and Diane Cooper
}

\begin{abstract}
Background: In the lives of women, puberty is marked by the onset of menarche. From this stage onwards until menopause, reproductive health and menstrual hygiene are important aspects of women's lives. In Zambia's Western Province, the natural process of menstruation is a taboo and dealt with secretly. Information and knowledge about menstruation and menstrual hygiene among adolescent girls is inadequate. This paper explores the factors influencing the understanding, experiences and practices of menstrual hygiene among adolescent girls in Mongu District, Western Province of Zambia.

Methods: An explorative study design was used by means of six focus group discussions conducted with 51 respondents, aged 13-20 years, from three secondary schools. Their age at menarche was 11-15. For data analysis thematic content analysis was used.

Results: The paper shows that the girls suffer from poor menstrual hygiene, originating from lack of knowledge, culture and tradition, and socio-economic and environmental constraints, leading to inconveniences, humiliation and stress. This leads to reduced school attendance and poor academic performance, or even drop outs, and ultimately infringes upon the girls' human rights.

Conclusion: To address these shortcomings, a 'super setting approach' is recommended, in which a Health Promoting School could improve the girls' individual and group needs, and a community setting which would address the broader socio-economic, cultural and environmental conditions. This would enable creating a supportive environment for the girls to manage their periods. To successfully utilize the approach, all stakeholders (parents, teachers, children, governments and communities) should cooperate to generate context-specific solutions for creating safe menstrual care, and better and dignified conditions for adolescent girls. Therefore, this calls for comprehensive, strident advocacy for policy changes at national level, and mediation and involvement at community level. (Global Health Promotion, 2018; 25(1): 54-62)
\end{abstract}

\section{Introduction}

Globally about $52 \%$ of women are of reproductive age, which means that menstruation is part of their lives (1). Puberty, marked by the onset of menarche, is recognized as an important stage, marking the transition from girlhood to womanhood $(2,3)$. From this stage until menopause, reproductive health and basic menstrual hygiene practices are important aspects in their lives (3). In many 
developing countries, however, including Zambia, the natural process of menstruation is dealt with in secrecy (4-6). This is due to taboos related to sexuality and reproduction, described by Warenius et al. (4) as gender norms and values related to culture and religion; norms that are influential barriers to communication on reproductive health issues among adolescents in Zambian society.

As a result, not much attention is paid to adolescent girls' specific needs as regards menstruation, despite the fact that to do so would establish a good foundation for their physical and mental well-being and their ability to cope with the demands of reproductive health later in life $(1,3,7)$. Such disregard can, and often does, lead to stress originating from soiled garments and bad odor and hence shame, as well as physical health problems such as urinary tract infections, pelvic inflammatory diseases and vaginal thrush (7-10). In addition, disregarding girls' specific needs for information about reproductive health can lead to gynecological complications, including HIV and other sexually transmitted diseases, and unwanted pregnancies which may later lead to unsafe abortions (11).

However, the consequences of this disregard are significantly more far-reaching, extending beyond the girls' health and hygiene, affecting their dignity, their schooling, educational careers and subsequent opportunities to lead fulfilled lives. This has been demonstrated in Africa and Asia where it is commonplace for girls to stay at home when menstruating rather than being exposed to the wide-ranging sources of accompanying stress (9,11-13). This, together with the fear of not doing well academically, has resulted in girls terminating their school careers (11,14-17) with negative life- long consequences.

Lack of information and the culture of silence in certain ethnic groups in Africa also results in menarche being regarded as an indicator that a girl must stop attending school and get ready for marriage. This has been described by Sommer (16) in his study of menstruation and schooling in Tanzania, where social pressure is employed to force girls who have reached reproductive age to enter into early marriages, meaning that they have to quit school. The study also highlighted how schools can be gender-discriminatory institutions and hence obstacles to girls' academic careers (16).

Yet these consequences are not inevitable, as the experience of puberty in more developed contexts demonstrates (2). With the appropriate support and resources at the individual, cultural and structural levels, puberty can and should be a natural biological function $(1,2)$.

\section{Potential strategies for support}

Health, hygiene, and sexual and reproductive health are recognized areas for health education interventions in schools, providing information to enable girls to make informed choices, and there are favorable reports about this approach in relation to preparing girls for menstruation (18). However, while valuable, this focuses primarily on individual behavior change, rather than the context in which girls live. 
Significantly more challenging are approaches that address the wider socio-economic and cultural factors that impact on the girls during their menstrual periods. There are several health promotion theories and approaches that could be adopted to address this broader focus.

As highlighted by Lohrmann (19), these include Bronfenbrenner's ecological systems theory, which argues that a child's development is affected by multiple layers of interacting influences, useful for exploring the impact of the environment on adolescents, their immediate family/community and the societal landscape. Also useful is the determinants rainbow developed by Dahlgren and Whitehead (20), which provides a reminder in any situation to look beyond the individual to the range of wider community, social, cultural and economic determinants.

An important means to encompass the above is the settings approach which has grown out of the principles and approach of the Ottawa Charter for Health Promotion(21), and has asits focus the place or social context in which people engage in daily activities. This includes the environmental, organizational and personal factors that interact to affect health and well-being (22), along with strategies to address the inherent tensions and differences in priorities between the different partners. It also supports working with communities $(23,24)$, including providing support, relevant information, and building their capacity to advocate for improved facilities. A Health Promoting School (24) is ideally placed to make a valuable contribution to adolescents, focusing beyond the individual to adopt an integrated approach that brings together different elements within the school itself and a collaboration with external stakeholders. Added to this is the more recent concept of a Super Setting (25), described as a coordinated intervention aimed at creating synergies across different settings in a coordinated manner to achieve a more comprehensive intervention.

With these considerations in mind, the study described aimed to explore the factors influencing the understanding, experiences and practices of menstrual hygiene among adolescent girls in secondary schools in Mongu District, Zambia.

\section{Methods}

\section{Study design}

An explorative qualitative study design was used to look into the perceptions, practices and experiences of girls associated with menstruation. This design was appropriate, providing the opportunity to gain an in-depth understanding and insight into the factors impacting on the girls' experiences.

\section{Study participants}

The study population consisted of 51 girls aged 13-20, in grades 9 and 12 in three secondary schools in the Mongu District. All had reached menarche. The schools were selected due to their location (rural, urban and peri-urban), and varied characteristics (government and mission schools, co and non-co-education) to enable the provision of wide-ranging views and experiences. The exclusion criteria were girls who had not reached menarche, were pregnant 
or had given birth, the latter being excluded as their circumstances were different due to information and sanitary materials given to them during ante-natal clinic visits.

Purposive sampling was adopted, selecting participants who could provide rich information about their experiences. The girls were divided into two groups: one comprising 13-15-year-olds (without much experience), and the other girls aged 16-20 years (with more experience). The separation into age groups was aimed at helping the 1315-year-old girls to discuss their experiences more freely amongst themselves and with the researcher than would have been possible if they had been merged with the older girls, who might have dominated or intimidated them.

\section{Data collection}

The main tool was focus group discussions (FGDs), preferred to individual interviews as it created an environment for exchanging views and experiences and sharing intimate details. There were six FGDs, two in each school, each comprising eight to ten respondents. The FGDs were conducted in both English and in the local language (Lozi) with the researcher as the moderator, and they ranged in duration from 1 to 2 hours. A research assistant provided support with tape recording, transcribing and translating into English where required. Participants were identifiable by numbering them, which was useful in interpreting their comments and key views during the analysis. Notes were also taken during the FGDs of aspects such as body language, dominance of any participant and any awkwardness. The questions asked during the FGDs included how the girls learnt about menstruation, their experience of menstruation and how it affected them in their daily lives, and how they managed the practicalities of menstruation, including the use and disposal of pads or alternatives and cleanliness. They were also asked about attitudes and practice about puberty in the community; and their opinion on the culture and taboos regarding menstruation.

\section{Data analysis}

Thematic content analysis was used, where the texts were broken down into themes (26). After familiarization with the data, it was possible to extract key issues and the categories and sub- categories pertaining to the girls' experiences of menstruation, and thereafter to code and to group and arrange the data as themes and sub-themes in accordance with the study objectives. Data analysis was done concurrently with data collection so that subsequent FGDs could build on the analysis of earlier discussions. The quotations selected from the raw data were typical of the views of participants interviewed and illustrate dominant themes.

\section{Ethical considerations}

Ethical approval was obtained for the study from the University of Western Cape, South Africa, and permission from the relevant Zambian district offices. Informed consent for participation was obtained from the girls and their parents/guardians and the girls were assured of confidentiality and anonymity. Given the sensitive nature of the study and the risk of upsetting any of the participants a psychosocial counselor from the Ministry of Health was available for support. However, in the event, this was not required. 


\section{Rigor}

To judge the quality of the study, the strategies of credibility and trustworthiness were applied. These included member checking, where the researcher re-addressed the participants to validate her findings; triangulation, comparing the data from each school and with the literature; reflexivity, where a detailed reflective diary was kept; and peer debriefing, checking data interpretations with the assistant researcher and the second author.

\section{Findings}

The descriptions of menstruation began with very immediate, practical concerns, providing a clear indication of the day-to-day realities for the girls. However, as the interviews progressed and the girls became familiar with each other and the interviewer, more underlying issues emerged. It became clear that menstruation was perceived as a state fraught with stress, humiliation and shame due to a lack of understanding by male peers and male teachers. These situations were compounded by cultural and socio-economic factors that the girls were unable to control. The most devastating impact noted, however, wastheimpactontheir education anditsimplications.

\section{Attitudes within the school}

While the majority of female teachers were supportive, the girls reported many cases of negative encounters from male teachers and male peers, ranging from the very simple, but traumatizing reactions to them staining their uniforms, to reports about a lack of understanding and even punitive action.

Some teachers don't understand us girls, the pain we go through during menstruation. I can give an example. If you are having your period and have period pains and so are notactive on that day, the teacher will say you are lazy because it is normal for women to have their periods every month and we should get used to it.

The girls reported horrific humiliating cases of classmates who, having accidentally messed themselves, were ordered by a male teacher to write a report about their misfortunes. You know, especially the male teachers, I don't think they understand that when you mess yourself up it is by accident.

They also reported living in permanent fear that their male classmates might detect their condition, either when they mess themselves accidently or a boy noticing a used pad in a girl's bag. These situations were used to ridicule the girl:

The boys ... will be laughing ... wherever you pass, they will be ... mocking and talking about you (being)... careless and dirty. Therefore ... we are affected psychologically and become stressed and always live in fear leading to low self-esteem, hence our school performances go down. Some even stop coming to school.

\section{The impact of culture and traditions}

The explanation given for this treatment was the Zambian culture of stigmatizing issues related to menstruation, reproductive health and sexuality which prevent them being discussed. This 
lack of awareness was therefore not restricted to the males. The girls also had scant awareness of menstruation and the female fertility cycle prior to the menarche. Furthermore, what they did know was often based on information from friends, who are often misinformed:

Before I started menstruating I didn't know about menstruation ... this issue is very secretive; it is not supposed to be discussed with young ones who have not yet started menstruating.

This left them frightened and confused when seeing menstrual blood for the first time:

I was scared and terrified as I did not know what was happening to me.

Those with some knowledge about menstruation prior to the menarche only knew about it as a biological event. They were not prepared for the extent to which menstruation would affect their lives, either in the short term in relation to their day-to-day management of their periods, or in the long term, in relation to its impact on their self-worth, their education and their futures in general.

To add to their distress, Lozi tradition includes an initiation process that the girls are required to conform to at the onset of the menarche. This was particularly stressful as it included being kept in isolation away from family and friends for between 3 weeks and 6 months. None of the girls liked it; they all found it humiliating and traumatic. Comments included:

When a girl is undergoing initiation, she is not supposed to be seen in public. If you are going out, you cover yourself with material so that your face is not seen. When people notice that your face is covered they know that you are undergoing initiation, which is very embarrassing and shameful. It becomes worse if your schoolmates, especially boys, notice you, they will be laughing and teasing you.

The initiations also led to them having to quit friendships with girls younger than themselves as they are not permitted to reveal what they have been taught to the younger girls:

You lose some of your old friends ... now you are grown up; you should not play or mix with girls who have not started menstruating.

They are also required to change their dress code in order not to reveal too much of their body shape, which might be seen as sexually tempting:

You are not allowed to wear short dresses, skirts or trousers, (you must) always move with a chitenge (traditional cotton scarf) wrapped around your waist (laughter).

An emotionally distressing focus of the initiation was the preparation for marriage, which included being a housewife, and about how to please their futurehusbands in bed:

Elders say you are now a woman; you are no longer a little girl you can even get married. 
For many this leads to early marriages and pregnancies, against Zambian law for those under 18 but silently tolerated in rural areas. The consequences of unwanted pregnancies were described as follows:

Other girls when they become pregnant try to abort and risk dying in the process. When the school authorities find out that you are pregnant or have aborted, you are chased out of school.

To add to their distress, the initiation process is concluded with a ceremony which announces that the girl now has reached the stage of being marriageable:

Your family will arrange for an overnight ceremony where they play drums ... (and drink and) dance the whole night.

The implications on their schooling were also devastating. As the initiation generally results in the girls being taken out of school for weeks or even months, it was seen as a threat to their academic performances. According to the girls, the schools do not provide any help with this, and many girls are unable to catch up with what they missed. As a result, many dropped out of school altogether.

\section{The implications ofpoverty}

Poverty emerged as a very significant barrier to the girls' ability to cope with menstruation. Most girls could not afford to buy sanitary pads.

Our parents struggle to meet school fees. So money for pads is not a priority.

Hence they resort to using old clothes and blankets torn into rags as these materials can be reused many times, and sometimes toilet paper as means of protection. Transportation presents a further important poverty-related problem. Those living far from school reported that they cannot afford the bus fares and so have to walk many hours, which they found particularly burdensome on the days they were experiencing heavy flows. This was further aggravated by having to walk long distances with a rag as pad, as this causes friction.

To the personal and family's poverty must be added the structural and environmental poverty of girls' homes and schools. First and foremost, they reported that water supply in schools and at home is inadequate. This was particularly stressful and inconvenient during their periods as they have to walk long distances to access water, and store it in buckets for when needed. It was also reported that there are cases when there are no washing facilities at the classroom blocks at the boarding schools, or that these are not functioning; hence they must walk (up to $700 \mathrm{~m}$ ) to the dormitories to change their menstrual cloths during classes. This is while lessons continue, once again wasting time that should be dedicated to studying. Another nuisance is that they do not have access to laundry lines to hang their pants or used 
rags, as girls are not supposed to expose their underwear, let alone their menstrual materials. So they dry these under their mattresses.

Keeping clean was also noted as a major concern. The bathrooms at the boarding schools were communal, with shared showers precluding any privacy. To compensate for this, the girls who are menstruating use the bathrooms when nobody is around, interrupting their sleep in search of privacy.

Water is a problem at this school; one has to wake up very early in the morning to go and draw water for bathing. It is very difficult to maintain a high standard of hygiene, maybe you only wash once a day and that's at night.

I wake up very early... and bath before others wake up ... then go back to sleep.

For those using sanitary pads there was the problem of a lack of sanitary disposal facilities, so they usually wrap used pads in old newspapers, which they hide in their school bags to dispose of after classes. Or alternatively, they avoid school altogether.

\section{Discussion}

The findings of this study demonstrate that menstruation, which is supposed to be a normal biological process, can turn into a threat to girls' social, physical and mental wellbeing, their schooling, and their future careers. It also shows that while each aspect has a significant impact in its own right, in combination, the implications are overwhelming. The FGDs provided a unique opportunity for the girls to talk openly and to share information about how shocking and discomforting the experience of coming of age through menstruation had been for them- experiences not normally discussed. The laughing and nodding in agreement by the girls during the FGDs, which at times disclosed stressful and embarrassing circumstances, added to their openness, and consequently, the depth of the discussions.

The Lozi society of Zambia's Western Province, the site of the study, is more conservative than most other Zambian communities. This means that they follow their tradition more strictly, including the belief that discussing bodily functions is taboo. This particularly relates to women's issues, including menstruation, where denying girls of pre-pubertal age the opportunity to understand the changes they will soon face results in misleading perceptions and attitudes among both girls and males (boys and men) they come in contact with. Yet despite the conservatism of the Lozi, this situation is not unique. There are several societies in other countries described in the literature which share the same culture of secrecy and rituals $(4,6,11-13)$, with similar consequences.

While the source and manifestation may have differed, the girls experienced discrimination both at home and in school. Gender discrimination at home was evident in not being allowed to mention menstruation in the presence of males, and being restricted in demonstrating any visible aspect of menstruation. At school the girls reported that they were intimidated and 
embarrassed by the treatment they received from their male teachers and peers who teased, mocked and humiliated them. As with experiences of girls elsewhere $(9,11,13)$, they therefore often opted to stay at home when menstruating rather than exposing themselves to this harassment. Another common theme described in both the literature and the current study was the lack of preparation for menstruation $(3,16)$. Neither parents nor teachers provide information to pre-menarche girls or psychological support when they start menstruating, at the time they need it most. These are challenging moments for girls to be faced by them alone.

Of particular significance in the current study was the embarrassing and intimidating experience of the traditional initiation ceremonies undergone by Lozi girls. For many the most devastating aspect was the disruption to their education. Participants expressed fear of not doing well academically as a result of missing lessons during initiation. This can take up to 6 months, and respondents reported that almost 50\% of the girls drop out of school as a result. The initiation process also gave a contradictory message. On the one hand, as 'grown up women', they had to adopt appropriate decorum, such as changing their mode of dress to prevent them being seen as sexually provocative, and avoid mixing with boys. Yet, at the same time, the initiation placed a strong emphasis on introducing the girls to sexual practices and readiness for marriage. This often encourages early sexual debuts, with the consequent exposure to health risks such as HIV, sexually transmitted infections, teenage pregnancy and early marriages (16). Once again these practices are not unique, with examples including puberty rituals in Malawi (11), and early marriages in Tanzania (16) and South India (8) resulting in early termination of the girls' schooling with negative life-long consequences.

Cultural stresses related to menstruation were compounded by the impact of the socioeconomic determinants of both personal and structural poverty. Despite their understanding of the health implications of poor menstrual hygiene, the structural poverty at home and at school meant the girls were unable to attend to their basic needs. These multiple impacts of poverty have been highlighted in many studies relating to health, hygiene and menstruation, especially in educational institutions $(11,16)$ which have been shown to undermine girls' privacy, and consequently, results in a fundamental infringement of their human rights $(5,16)$.

Inevitably, all studies have their limitations. In this instance, it includes the constraints of what was a small qualitative study which, while exploring the problems in-depth, cannot be seen to be representative. In addition, interviews with teachers, families and male peers would have been valuable, adding depth through alternative perspectives. For these reasons, further investigation in the area of reproductive health and menstrual hygiene in schools in diverse contexts is warranted.

\section{Implications for health promotion}

Despite these limitations the study has provided valuable insights into the complexity of the problems facing girls when they begin menstruating, issues that are not adequately recognized or addressed. These provide a significant challenge for health promotion. An 
integrated ecological framework, described, among others, by Lohrmann (19), would therefore be beneficial, enabling multiple levels of personal, organizational and environmental factors to be considered. Also important would be a focus on empowerment of both the families and communities to advocate for the changes to take place $(23,24)$.

The most challenging, and yet most crucial aspect to address would be the local cultural practices. While recognizing the role a strong community plays in providing social cohesion and support, in some cases traditional practices can exacerbate problems. The study has demonstrated how the cultural taboos and initiation ceremonies have detrimental implications for the girls' health, their education and the awareness of the male communities that they live amongst. In order to address these sensitive issues, an approach is needed that is rooted in the community and mindful and respectful of the community beliefs (23). Challenges to the local traditions need to come with support from the communities if they are to be effective. This inevitably requires time and sensitivity to generate a foundation of mutual trust and respect between the community members and health promoters. The literature provides examples of interventions where this has been achieved, where communities define the problems and seek solutions, encouraging people to speak out and engage in discussion harnessing traditions into positive practices (27). Drawing on community members, possibly younger women, and also female teachers to try and shift the cultural conservatism would be an essential part of the approach to enable sensitive interventions.

The second focus would need to be one that addresses the broad socio-economic constraints, both in the community and at schools. This would require a collaborative approach across government, both national and local, and non- governmental agencies to build the infrastructure that enables a more supportive environment for health (20). An example would be intersectoral policies and programs to improve the water and sanitation provision in the schools and in the community, and to develop financial support and poverty reduction measures.

Finally, and importantly, there would be a need for reproductive health awareness interventions, including menstruation, in schools and in the community at large, to prevent the stresses the girls encounter due to ignorance about both the biological changes and the attitudes of others. These, however, would only be effective if the overarching determinants described above were addressed.

A Super Setting is a useful approach to address these multiple layers of need (25). A Health Promoting School could improve the individual requirements of the girls by providing relevant information, policies and a general supportive environment for them to manage their periods optimally while at school. This could accommodate the awareness needs of both females and males, and address the more practical and structural aspects, from affordable access to sanitary towels to improved laundry and sanitation facilities. A wider community setting would be appropriate to address the 
issues that extend beyond the scope of the school, that is, the restrictive traditional practices and the adverse living conditions and overall poverty within the population. These would inevitably require a more complex integrated longer-term approach that included legislation, policy development and active involvement of stakeholders and communities at all levels. While this would be more difficult to achieve, it would be essential to enable realistic and sustainable change.

How does this relate to the human rights of the girls? The link between health education and human rights has been well recognized, as has the loss of dignity among vulnerable people due to the state and its institutions' failure to recognize or respond to their needs (28). Conversely, the promotion and protection of health are inextricably linked to the promotion and protection of human rights and dignity. Adopting measures that address the wider determinants of health and empower communities will therefore be part of the journey towards promoting the human rights of the girls. These are not an optional extra. Rather, they should be a fundamental part of a health promotion program that explicitly recognizes and seeks to address a human rights approach to health. It is also a fundamental contribution to the education rights and therefore future opportunities of adolescent girls.

\section{Conclusion}

The study sought to explore the factors impacting on girls' experiences during the menarche, and the impact this had on their health and their education. What became evident was that the combination of a lack of information, cultural taboos and poverty, both personal and within the schools, had a devastating impact on the girls, limiting their education, and consequently impacting on their human rights. The findings in this study are similar to those from other parts of Africa and Asia $(4,6,8,11-13,16)$.

There are existing health promotion strategies for addressing many of these complex issues. Yet the problem persists. The value of this study, along with others raising these issues, is to highlight the extent of the impact of these separate but compounding factors, with the view to encouraging a more comprehensive approach that addresses the wider determinants of menstruation. Based on our research, we recommend a 'Super Setting approach' to address both the individual and the broader socioeconomic, cultural and environmental conditions. This approach should not be limited to Zambia's Western Province, but should be effective for the whole country, as well as being relevant for other countries in which girls are similarly disadvantaged. In order to successfully utilize the approach, all stakeholders, i.e. parents, teachers, children, governments and communities should cooperate to generate context-specific solutions for creating safe menstrual care and a better and dignified future for adolescent girls. This calls for a clear assessment of the problem, and strident advocacy for policy changes at a national level and mediation and involvement at community level. 


\section{Declaration of conflicting interests}

None declared.

\section{Funding}

This research received no specific grant from any funding agency in the public, commercial, or not-for-profit sectors. 


\section{References}

1. House S, Mahon T, Cavill S. Menstrual hygiene matters: A resource for improving menstrual hygiene. Available from: www wateraid.org/mhm. (accessed January 2, 2014).

2. Dhingra R, Kumar A, Kour M. Knowledge and practices related to menstruation among tribal (Gujjar) adolescent girls. Stud Ethno Med. 2009; 3: 43-48.

3. Nagar S, Aimol RK. Knowledge of adolescent's girls regarding menstruation in tribal areas of Meghalaya. Study Tribes Tribals. 2011; 8: 27-30.

4. Warenius L, Pettersson KO, Nissen E, Hojer B, Chishima P, Faxelid E. Vulnerability and sexual and reproductive health among Zambian secondary school students. Cult Health Sex. 2007; 9: 533-544.

5. Patkar A, Bharadwaj S. Menstrual hygiene and management in developing countries. Taking stock. Mumbai: Junction Social; 2004. Available from: www.mum.org/menhydev.htm. (accessed December 29, 2013).

6. Mahon T, Fernandes M. Menstrual hygiene in South Asia. A neglected issue for WASH (water, sanitation and hygiene) programmes. Gender Dev. 2010; 18: 99-113.

7. Narayan KA, Srinivasa DK, Pelto, Veerammal S. Puberty rituals, reproductive knowledge and health of adolescent girls in South India. Asia Pacific Pop J. 2001; 16: 2.

8. Ministry of Health. Reproductive Health Policy. Reproductive Health Unit, Lusaka. Zambia; 2000.

9. Omidvar S, Begum K. Factors influencing hygienic practices during menses amongst girls from South India. A cross sectional study. Int J Collab Res Intern Med Public Health. 2010; 2: 411-423.

10. Oche MO, Umar AS, Gana GJ, Ango JT. Menstrual health: The unmet needs of adolescent girls' in Sokoto, Nigeria. Sci Res Essays. 2012; 7: 410-418.

11. Pillitteri PS. School menstrual hygiene management in Malawi: More than toilets. Sanitation and hygiene applied research for equity. 2011. Available from: http://www.wateraid.org/uk/what_we_do/how_we_work/equity_and_inclusion /10745.asp (accessed May 12, 2014).

12. Kirk J, Sommer M. Menstruation and body awareness: linking girls' health with girls' education. Royal Tropical Institute (KIT), Special on Gender and Health. 2006; 1-22, Amsterdam, The Netherlands.

13. Shanbhag RD, Shilpa R, D’Souza N, Josphine P, Singh J, Goud BR. Perceptions regarding menstruation and practices during menstrual cycle among high school adolescent girls in resource limited setting around Bangalore city Karnataka, India. Int J Collab Res Intern Med Public Health. 2012; 4: 1353-1362.

14. Ten VTA. Menstrual hygiene: A neglected condition for the achievement of several Millennium Development Goals. Europe External Policy Advisors. 2007. Available from: http://www.eepa.be/wcm/dmdocuments/BGpaper_ Menstrual-Hygiene.pdf (accessed March 19, 2014).

15. WaterAid. Is menstrual hygiene and management an issue for adolescent girls? A comparative study of four schools in different settings of Nepal. 2009. Available 
from: $\quad$ www.indiahabitat.org/qefl/link/Practices/wa_nep_mhm_rep_march20o9.pdf. (accessed March 20, 2014).

16. Sommer M. Menstruation and school attendance in Sub-Saharan Africa. Girls' experiences of menstruation and schooling in urban and rural Kilimanjaro, in Northern Tanzania. 2008. Available from: http://2008youthconference.blogspot.com/2008/04/menstruation-and-schoolattendance.htm. (accessed December 30, 2013).

17. Lawan UM, Nafisa WY, Aisha BM. Menstruation and menstrual hygiene among adolescent school girls in Kano, North-western Nigeria. Afr J Reproduct Health. 2010; 14: 201-207.

18. Fakhri M, Hamzehgardeshi Z, HajikhaniGolchin NA, Komili A. Promoting menstrual health among Persian adolescent girls from low socioeconomic backgrounds: a quasi-experimental study. BMC Public Health. 2012; 12: 193.

19. Lohrmann DK. A complementary ecological model of the coordinated school health program. J School Health. 2010; 80: 1-9.

20. Dahlgren G, Whitehead M. Policies and strategies to promote social equity in health. Institute for Future Studies: Stockholm (Mimeo); 1991.

21. Green LW, Poland BD, Rootman I. The settings approach to health promotion, in Settings for Health Promotion, Linking Theory and Practice, Poland DB, Green LW, Rootman I (eds) Thousand Oaks, CA: SAGE Publications; 2000; 1: 1-43.

22. Nutbeam D. Health promotion glossary. Health Promot Int. 1998; 0113: 4.

23. Gryboski K, Yinger NY, Dios R, Worley H, Fikree FF. Working with the community for improved health. Health Bull. 2006; 3. Washington, DC: Population Reference Bureau.

24. Laverack G, Nastaran K, Samdal O, Rowling L. Theoretical and empirical base for implementation components of health-promoting schools. Health Educ. 2011; 111: 367-390.

25. Bloch et al. Revitalizing the setting approach - Supersettings for sustainable impact in community health promotion. Int $J$ Behav Nutrition Phys Activity. 2014; 11: 4-15.

26. Terre Blanche M, Durrheim K, Kelly K. Section 3 - First steps in qualitative data analysis. Research in Practice. Applied Methods for the Social Sciences. Cape Town, South Africa: UCT Press; 2006. pp.321-370.

27. UNICEF. Changing a harmful social convention. Female genital mutilation/cutting. UNICEF Innocenti Research Centre, 2008.

28. Mann JM, Gostin L, Gruskin S, Brennan T, Lazzarini Z, Fineberg HV. Health and human rights. The sident and Fellows of Harvard College. 1994; 1: 6-23. 